\title{
Visual Graphic Analysis of the Complex Network of Expressways in Sichuan Province
}

\author{
Lingli Tang ${ }^{1, a}$, Kang $\mathrm{Li}^{2, \mathrm{~b}}$ \\ ${ }^{1}$ Department of Economic Management, Sichuan Vocational and Technical College of \\ Communications, Chengdu, 611130, China \\ ${ }^{2}$ Institute of Transport, Department of Transportation Engineering, Sichuan Vocational and \\ Technical College of Communications, Chengdu, 611130, China \\ a1249922109@qq.com, b876286380@qq.com
}

\begin{abstract}
Keywords: complex network, expressway, Sichuan province, Gephi
Abstract. The formed complex network of expressways in Sichuan province was visualized and studied by utilizing complex network theory and Gephi software to analyze recent expressway data of Sichuan Province. The main characteristics and existing problems of the network were studied, for the purpose of providing a new perspective and decision-making reference to the transportation-related governmental departments for transportation network planning, infrastructure construction and scientific management.
\end{abstract}

\section{Economic Development and Traffic Achievements in Sichuan Province}

Under the "New Normal" policy of Chinese economy, which turns from high speed to medium speed growth, the Sichuan Province of China achieved production value of 2,835.7 billion yuan (up by $8.5 \%$ ) in 2014, and the index of per capita disposable income of urban residents increased by $9 \%$ (See Fig. 1).

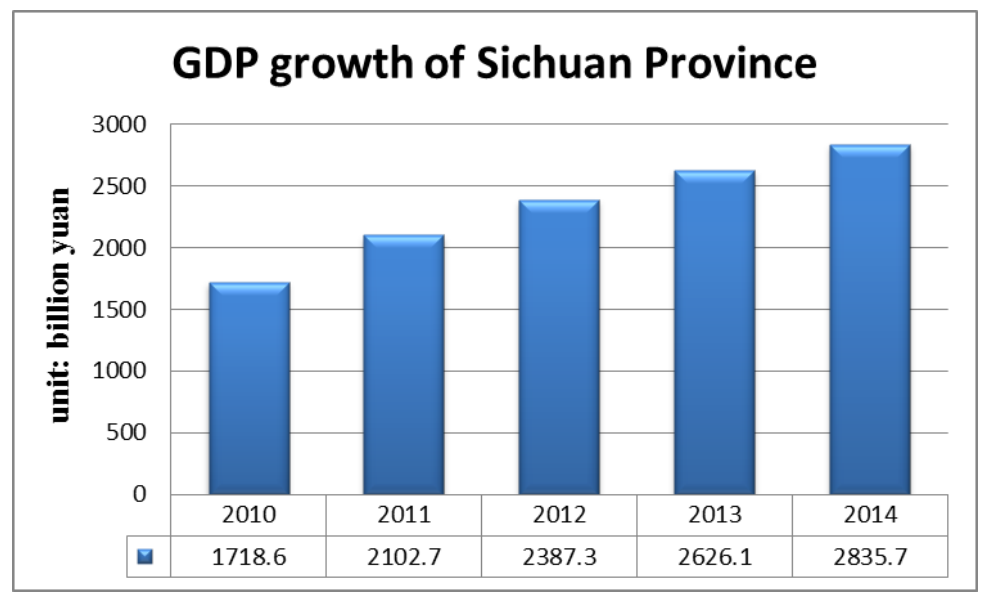

Fig. 1 GDP growth in Sichuan Province 1

In terms of transportation, Sichuan province completed cargo turnover of 247.4 billion ton-kilometers, and passenger turnover of 158.4 billion person-kilometers in 2014. Its railway operating mileage reached $3,958 \mathrm{~km}$, annual inland port container handling capacity achieved 441 thousand TEU, and expressway traffic mileage attained $5,510 \mathrm{~km}$ (See Figure 2). This lead to the formation of integrated transport hub in west China, and expressway network were coming into being."Shudao Hard" history is gradually disappearing.

With rapid development of traffic construction in Sichuan Province, the mileage of expressway is increasing, and the complex network is gradually formed. So in this paper, we utilize the visual graphic analysis tool-GephiI to analyze topology and characteristics of the formed expressway complex network, study its disciplines and change mechanism, propose some scientific management

${ }^{1}$ Data sources: Statistical yearbook of Sichuan province -2014 
and decision-making advice to the relevant governmental bureaus of planning, construction, and management.

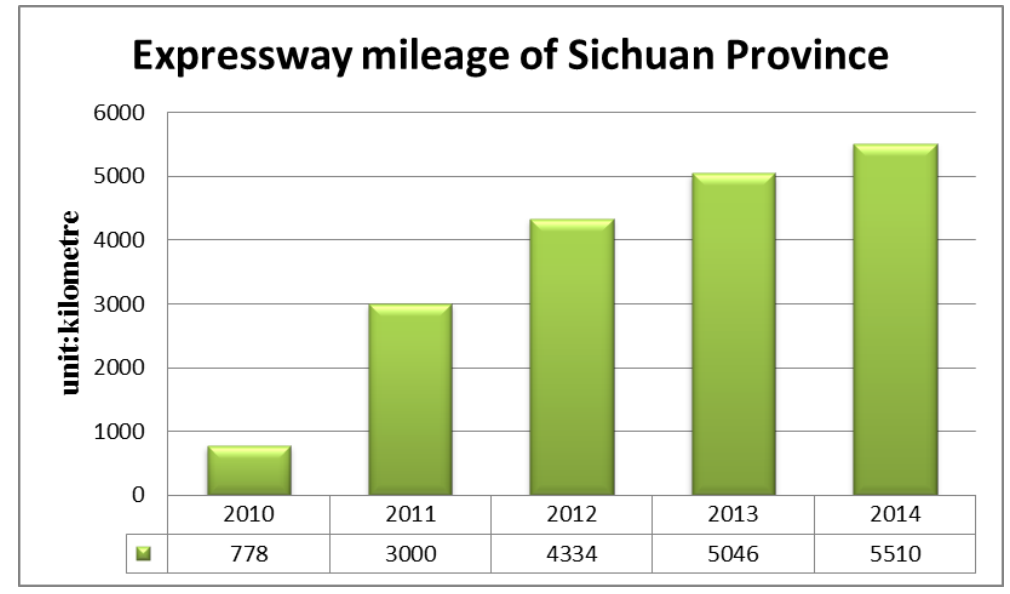

Fig. 2 Expressway mileages in Sichuan Province ${ }^{2}$

\section{Introduction of the Complex Network Analysis Tool-Gephi}

Gephi is a cross-platform open-source free software based on JVM. It was jointly researched and developed by multinational engineers and scientists, mainly for the analysis of a variety of complex networks and systems. Starting in 2008 in France, Gephi has been supported, protected and promoted its continuous development by non-profitable, specialized agencies-the Gephi Alliance.

Gephi is used to visualize and detect the interaction between dynamic and hierarchical graphs of various networks and complex systems, such as: association analysis, data analysis, social network, biological network and other complex network analysis. In addition to generate visual graph of a complex network based on the input data, Gephi can also automatically calculate and generate topological network characteristic parameters analysis diagram corresponding to the complex network. This function is particularly important to the complex relationship analysis of massive data under current "big data era" background.

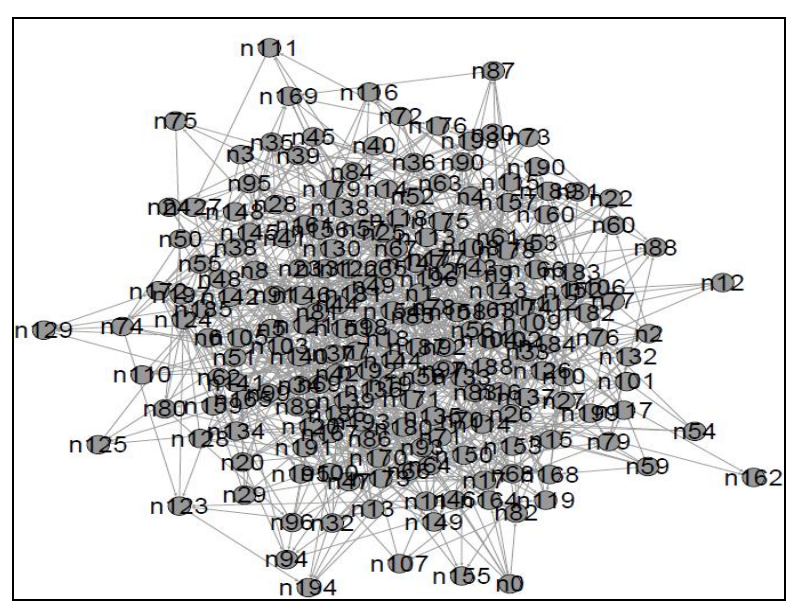

Fig. 3 Randomly generated Gephi diagram example with 100 nodes 


\section{Visual Graphic Analysis of the Complex Network of Expressway in Sichuan Province based on Gephi}

With rapid globalization of economy and development of science and technology, the transportation system, which is a supporting system of social and economic system, is becoming more and more complicated. At the same time, emergence and evolution of the complex network theory provides a scientific and systematic approach to study the emerging complex systems.

Basic Expressway data in Sichuan Province. The data used in the paper is obtained from the publicly released highway network data of Sichuan province (http://www.scjtcx.cn/). Network nodes are selected according to the given nodes and road networks in the webpage (see Tab. 1). Nodes on each highway are arranged according to the entrance and exit sites' order of expressways, whose details are given by Baidu data. Prefecture level city above is marked by its name directly.

Tab. 1 Sichuan provincial expressway data (open to traffic by the end of 2014)

\begin{tabular}{|c|l|}
\hline & $\begin{array}{l}\text { G42 Guanglin expressway; G42 Lindian expressway; G42 Nanguang expressway; G42/G75 } \\
\text { Chengnan expressway; G4201 Chengdu Ring expressway; G5 Guangbei expressway; G5 }\end{array}$ \\
Pantian expressway; G5 Xipan expressway; G5 Yalu expressway; G5 Luhuang expressway; \\
G5/G93 Chengmian expressway; G5/G93 Chengya expressway; G5/G93 Mianguang \\
expressway; G65 Dashan expressway; G65 Dayu expressway; G75 Guangganexpressway; G75 \\
express \\
$\begin{array}{l}\text { Guangnan expressway; G75 Nanyu expressway; G75/G42 Chewngnan expressway; G76 } \\
\text { Longna expressway; G76 Naqian expressway; G76/G85 Chengyu expressway; G85 Neiyi } \\
\text { expressway; G85 Yishui expressway; G93 Luyi expressway; G93 Miansui expressway; G93 } \\
\text { Yiyu expressway; G93/S11 Suiyu expressway }\end{array}$ \\
\hline $\begin{array}{l}\text { Sichuan } \\
\text { province }\end{array}$ & $\begin{array}{l}\text { S1 Chengmian double expressway; S1Chengpeng expressway; S11 Suinei expressway; S2 } \\
\text { expressway; S20 Guangba-Guangnan connecting expressway; S4Chengzilu expressway; } \\
\text { S6Chengdu airport expressway; S7 Chengle expressway; S8 Chengwenqiong expressway; S8 } \\
\text { Qiongmin expressway; S9 Chengguan expressway; S9 Duwen expressway; S9Yingwen } \\
\text { expressway; SA56 Nanchong ring expressway; SB05 Suihui expressway }\end{array}$ \\
\hline & $\begin{array}{l}\text { XA45 North Chengdu exit expressway; Duwen expressway; Guangshan expressway; Leya } \\
\text { expressway; Xingcang satellite expressway; Yaxi expressway }\end{array}$ \\
\hline others &
\end{tabular}

The Gephi-based generated complex network of expressways in Sichuan Province. Considering balance of network size, feasibility of characteristics analysis, data acquisition and other factors, the data of Sichuan Expressway Network was selected from the open websites. Network nodes were selected according to the original data of the networks, and the generated graph based on Gephi is exhibited in Fig. 4.

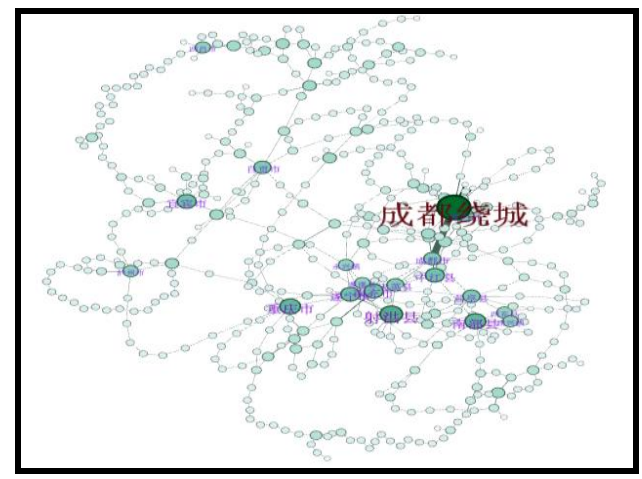

Fig. 4 Complex network of expressways in Sichuan Province 
It can be seen from the network diagram that there are totally 476 nodes, 588 sides in the expressway complex network of Sichuan province. In the formed complex network, Shehong County, South County, Suining, Nanchong, Yibin, Zhongjiang and other cities and counties, which have greater node degrees than the others, connect larger number of highways, and have relatively more smooth traffics. Due to the fact that almost every expressway connects Chengdu city by the ring expressway to reach center of the city, number of the Province expressways connecting Chengdu city in the figure is not high, but is the Chengdu ring expressway with the highest degree.

Visualization and Characteristic analysis of the complex network of Expressways in Sichuan Province. Application of Gephi visualization software, we can not only get the provincial highway complex network visualization graphics, but also can analyze and evaluate topology characteristics of the generated network automatically. This is the main reason for choosing Gephi as the analytical tool.

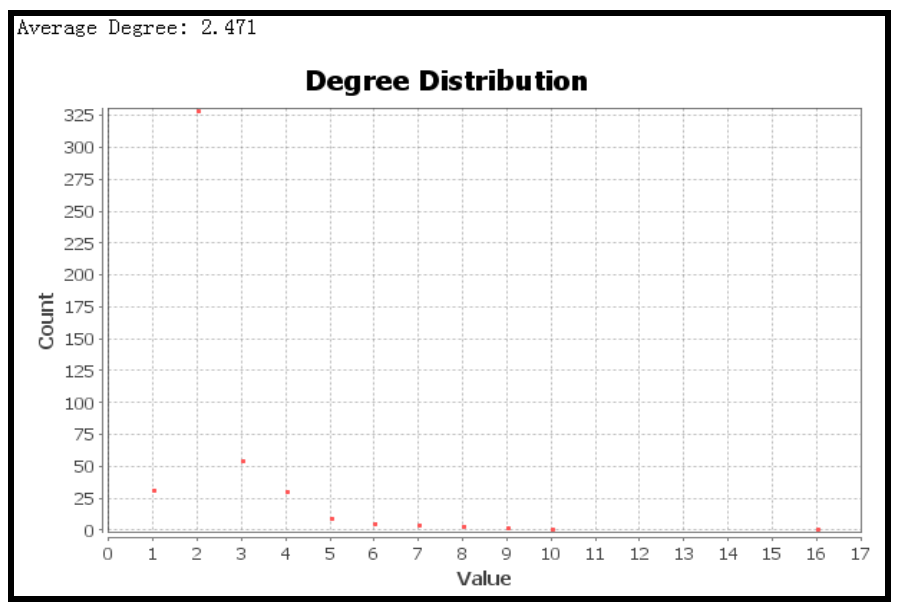

Fig. 5 Nodes' degree distribution of the expressway network

As can be seen from Fig 5, the biggest node degree value is 16, about 120 nodes' degree value are greater than 2, about 440 nodes' degree values are greater than 1, about 30 nodes' degree values are equal to 1, accounting for $6 \%$ nodes in the complex network of expressways of Sichuan province.In the whole network, there are $25 \%$ of the nodes' degree are greater than 2 , that means in the actual highway network, $75 \%$ of the toll stations' accessibility are high, which bear most of traffic volume in the province. This also shows that in recent years, highway constructions in Sichuan province enhanced the local transport level significantly.

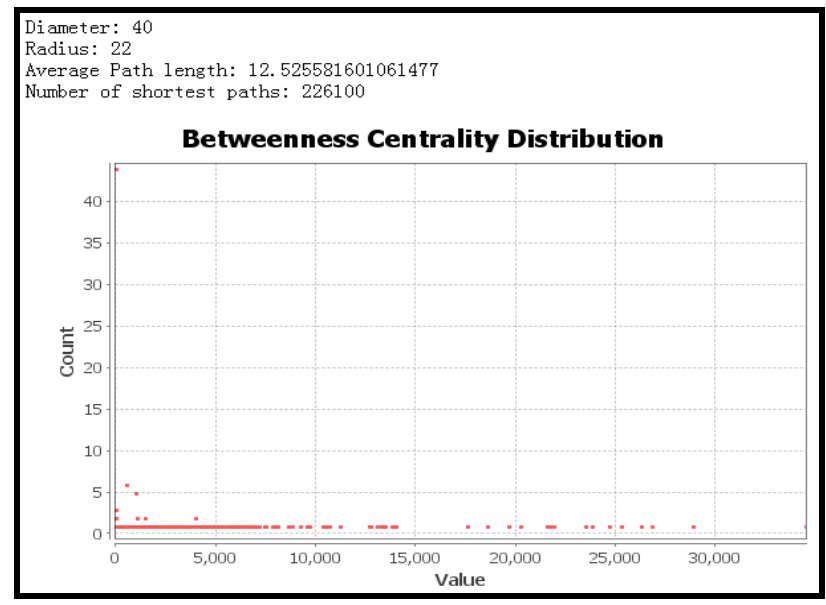

Fig. 6 Nodes' betweenness centrality distribution of the expressway network

As can be seen from Fig. 6, the average path length of the weighted network in Sichuan province is 12.53, which shows that the average shortest travel of any two stations in the network is 12.53 toll 
stations. At the same time, it shows that the region of Sichuan province is wide, especially in mountainous area, which makes the expressway in local area become the only access to the area. In addition, the diameter of the highway network in Sichuan province is 40 , which indicates that a travel from one end of the network to the other end includes at least 40 toll stations. It also shows that the highway network in Sichuan province connect some remote mountainous areas, which makes the network a long diameter.

As can be seen from figs. 7-8, sites with better closeness centrality in the figure 4 are located in Chengdu city and center location of its annex network. These nodes connect large number of roads relatively, whose accessibility is good. And its service scope is relatively large, so they can improve public travel convenience and cargo transport efficiency in the local area. At the same time, they also easily induce traffic congestion. Therefore, they need to be strengthened traffic management and guidance, limited vehicle traffic reasonably, to reduce the occurrence of traffic accidents. In addition, roads with high closeness centrality index are more reachable, have higher propagation velocity and larger influence area in the network traffic flow, thus have higher survivability. When congestion or traffic accidents appear, in order to reduce the extent of the impact to the whole road network, the scope of the accident can be quickly determined according to the sites' closeness centrality index, and measures can be taken to restore the traffic in the shortest possible time.

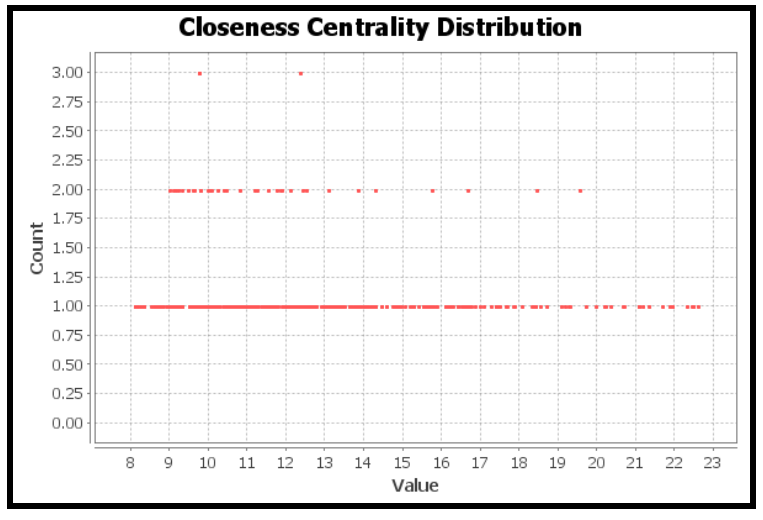

Fig. 7 Nodes' close centrality distribution map

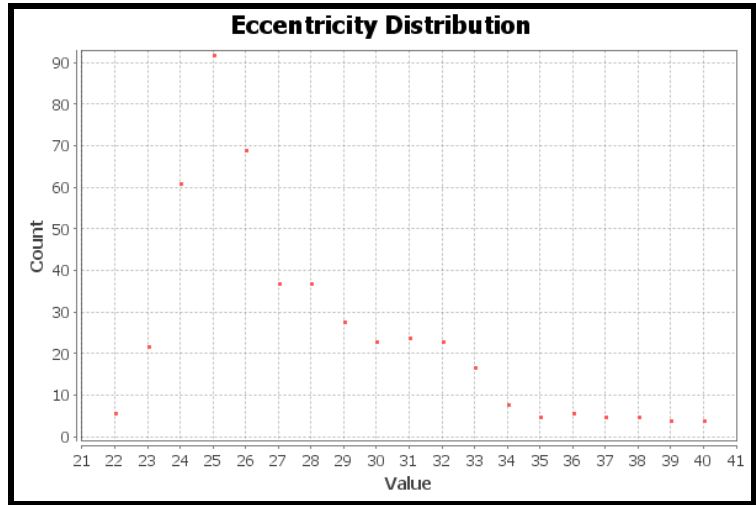

Fig. 8 Nodes' eccentricity distribution map

It can be seen from the figs. 9-10 that th expressway network of Sichuan province has higher degree of modularity (Different colors represent different modular regions, and the internal connectivity of the same modular area is better).

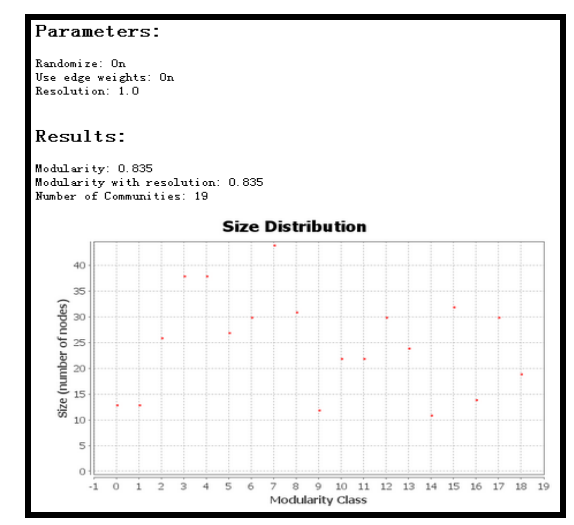

Fig. 9 nodes' modularity class distribution map

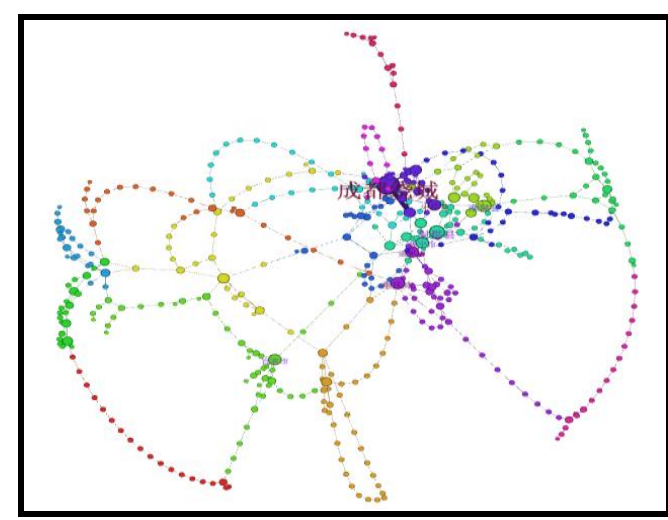

Fig. 10 layout of expressway network in Sichuan Province

\section{Countermeasures and suggestions}

From the above discussion of topology modeling and analysis results of the expressway network, the Expressways in Sichuan Province are mainly concentrated in Chengdu, Yibin City, Suining City, 
Guangyuan City, Shehong County, and South County, and transportation development is unbalance between north-south part and east-west part in Sichuan province. In the shortest path, important toll stations are relatively concentrated in the central area of Chengdu city. In the future, the highway construction needs to minimize the direct connection with the higher central index nodes to prevent further increase to the traffic flow. In this way, the traffic load of these important nodes can be reduced, and the safe operation of toll station nodes in the core area is guaranteed. But taking into account the transportation efficiency and connectivity of the whole network, and the necessity of connection with these high level of reachability, it is recommended to avoid the direct connection with these important nodes, arrange the connection with other neighboring nodes. It is necessary to consider the setting of the base ring, and reduce the traffic pressure of the node of the vehicle. This method can also effectively improve the speed of vehicle flow.

\section{Acknowledgements}

This work was financially supported by the Projects of Science and Technology Department of Sichuan Province (2011ZR0177 and 2011GZ0127), Projects of Department of Transportation of Sichuan Province ("Research on the intensive development strategy of road transportation in Sichuan Province" and 2015C1-1), and projects of sichuan vocational and technical college of communications(2014-580-03).

\section{References:}

[1] Work report of the people's Government of Sichuan Province in 2015 [EB/OL].http://www.sc.go v.cn/10462/10464/10797/2015/2/5/10326259.shtml, 2011-11-17/2015-04-10

[2] Sichuan public travel traffic information service system - Sichuan road network [EB/OL].http:// www.scjtcx.cn

[3] Sichuan Provincial Department of transportation website [EB/OL].http://www.scjt.gov.cn/10000/ 10001/10002/11475/index.shtml

[4] Road Transport Administration Bureau of Sichuan Provincial Department of transportation [EB/ OL].http://www.scyg.gov.cn/overview

[5] Highway Bureau of Sichuan Provincial Department of transportation [EB/OL].http://www.scgl.n et/10001/ index. shtml

[6] Sichuan Provincial Highway Administration Bureau (highway traffic law enforcement corps)[EB /OL]. http://www.scgl.net/10001/index.shtml

[7] China Statistical Yearbook 2014 [EB/OL].http://www. stats .gov.cn/tjsj/ndsj/2014/indexce.htm

[8] China highway statistical yearbook [M].China Statistics Press.2014

[9] Gephi Chinese tutorial, https://www.udemy.com /courses /search?q=gephi

[10] 09-2013 China expressway construction trends and investment outlook planning report [R].200 9.11

[11] Z. Gao, J. Wu, B. Mao, etc. Research on the complexity of transportation network and its relate d problems [J]. Transportation systems engineering and information, 2005, 5(2): 79—84

[12] X. Zhao, Z. Gao, H. Huang. Spatiotemporal modeling of complex networks [J]. Transportation systems engineering and information, 2006, 6(3): 53-57

[13] W. Zhao, H. He, Z. Lin. Research on the network nature of China's railway passenger transport network [J]. Journal of Physics,2006, 55(8): 3906-3911

[14] L. Yang, D. Qian. Research on the vulnerability of road traffic network [J] .Transportation sys tems engineering and information, 2011 (2) : 105-110

[15]Q. Liu, H. Chen, J. Zhou, etc. Study on invulnerability of expressway network based on complex network theory [J].Highway, 2014（6）:214-219

[16] P. Xiao. Complexity analysis of highway network structure in Guangdong Province [D] .Sout 
h China University of Technology, 2013 